\section{Can an automated \\ faecal classifier be used to identify and grade severity of diarrhoea from veterinary clinical narratives?}

\section{Amy Jackson, Jenny Newman, P-J.M Noble}

University of Liverpool, Neston, Cheshire, UK

\section{OBJECTIVES}

Practitioners use different terminology when documenting the consistency of diarrhoea described by the owner. A system that could automatically classify descriptions of diarrhoea documented in the clinical narrative would allow the severity and temporal patterns of diarrhoea to be studied without requiring explicit coding by the vet.

\section{METHODS}

The study population consisted of consultations collated within the SAVSNET database where the patient was a dog that had had at least one consultation for diarrhoea in the period from 22 ${ }^{\text {nd }}$ November 2013 to $10^{\text {th }}$ April 2016. The clinical narratives were examined to understand the adjectives and similes used by veterinary surgeons when describing faecal consistency and this was used to create a text-mining classifier consisting of a group of regular expressions written in Python.

\section{RESULTS}

The sensitivity of the faecal consistency grader was $96.88 \%$, where by the classifier found a faecal consistency matching that assigned during manual reading of the record. The classifier identified a faecal consistency in $61.23 \%(n=2577)$ of consultations within the study population. A faecal grade of $5(n=1544)$, reflecting watery diarrhoea, occurred in the largest proportion of animals where a grade could be identified.

\section{STATEMENT}

The study showed that an automated faecal grading system could be used to search for faecal consistencies in clinical narratives. This form of faecal grading system could be used to monitor progression of disease in populations of small animal patients and could be developed to identify specific phenotypes of gastrointestinal disease in the dog population.

\section{Assessing the utility of the neutrophil-to- lymphocyte ratio to differentiate canine inflammatory bowel disease from intestinal lymphoma}

\section{Camilla Hindar, Gerard McLauchlan, Timothy Parkin}

University of Glasgow, Glasgow, UK

\section{OBJECTIVES}

To assess the utility of the neutrophil-to-lymphocyte ratio (NLR) to differentiate between canine inflammatory bowel disease and canine intestinal lymphoma.

\section{METHODS}

A retrospective study was performed in dogs diagnosed with either inflammatory bowel disease or intestinal lymphoma. Cases were excluded if a pre-diagnosis haematology was not available, if they had received medications that may influence the NLR prior to the haematology sample being obtained, if they had undergone surgery within the 2 months prior to diagnosis, or if they had any concurrent illnesses identified during investigations. Cases included had a histological diagnosis of inflammatory bowel disease or intestinal lymphoma, or a combination of cytological diagnosis and ultrasonographic findings consistent with intestinal lymphoma.

\section{RESULTS}

Statistical analysis showed that dogs diagnosed with inflammatory bowel disease $(n=32)$ had a significantly lower NLR than those diagnosed with intestinal lymphoma $(n=17) \quad(p=0.001)$. In dogs with inflammatory bowel disease the mean and median NLR were 7.88 and 7.07, compared to 29.9 and 22.74 , respectively, in those diagnosed with intestinal lymphoma. An NLR threshold value of 14 (sensitivity of $82 \%$, specificity of $84 \%$ ) had a positive predictive value of $74 \%$ and negative predictive value of $90 \%$.

\section{STATEMENT}

The neutrophil-to-lymphocyte ratio (NLR) is a useful and cost-effective biomarker in aiding to differentiate between canine inflammatory bowel disease and canine intestinal lymphoma. 